\title{
Olig2-expressing Mesenchymal Stem Cells Enhance Functional Recovery after Contusive Spinal Cord Injury
}

\author{
Hwan-Woo Park ${ }^{1,2, *}$, Soonyi Oh ${ }^{1, *}$, Kyung Hee Lee ${ }^{3}$, Bae Hwan Lee ${ }^{4}$, Mi-Sook Chang ${ }^{1,5}$ \\ ${ }^{1}$ Laboratory of Stem Cell E Neurobiology, Department of Oral Anatomy, Dental Research Institute E School of Dentistry, Seoul \\ National University, Seoul, Korea, ${ }^{2}$ Department of Cell Biology, Myunggok Medical Research Institute, Konyang University College \\ of Medicine, Daejeon, Korea, ${ }^{3}$ Department of Dental Hygiene, Dongseo University, Busan, Korea, ${ }^{4}$ Department of Physiology, Brain \\ Korea 21 Project for Medical Science, Yonsei University College of Medicine, Seoul, Korea, ${ }^{5}$ Neuroscience Research Institute, Seoul \\ National University, Seoul, Korea
}

Background and Objectives: Glial scarring and inflammation after spinal cord injury (SCI) interfere with neural regeneration and functional recovery due to the inhibitory microenvironment of the injured spinal cord. Stem cell transplantation can improve functional recovery in experimental models of SCI, but many obstacles to clinical application remain due to concerns regarding the effectiveness and safety of stem cell transplantation for SCI patients. In this study, we investigated the effects of transplantation of human mesenchymal stem cells (hMSCs) that were genetically modified to express Olig2 in a rat model of SCI.

Methods: Bone marrow-derived hMSCs were genetically modified to express Olig2 and transplanted one week after the induction of contusive SCI in a rat model. Spinal cords were harvested 7 weeks after transplantation.

Results: Transplantation of Olig2-expressing hMSCs significantly improved functional recovery in a rat model of contusive SCI model compared to the control hMSC-transplanted group. Transplantation of Olig2-expressing hMSCs also attenuated glial scar formation in spinal cord lesions. Immunohistochemical analysis showed that transplanted Olig2-expressing hMSCs were partially differentiated into Olig1-positive oligodendrocyte-like cells in spinal cords. Furthermore, NF-M-positive axons were more abundant in the Olig2-expressing hMSC-transplanted group than in the control hMSC-transplanted group.

Conclusions: We suggest that Olig2-expressing hMSCs are a safe and optimal cell source for treating SCI.

Keywords: Olig2, Mesenchymal stem cells, Spinal cord injury, Transplantation

Received: August 25, 2018, Revised: October 3, 2018,

Accepted: October 3, 2018, Published online: October 31, 2018

Correspondence to Mi-Sook Chang

Laboratory of Stem Cell \& Neurobiology, Department of Oral Anatomy, Dental Research Institute \& School of Dentistry, Seoul National University, 101 Daehak-ro, Jongno-gu, Seoul 03080, Korea

Tel: +82-2-740-8628, Fax: +82-2-745-1906

E-mail: mschang@snu.ac.kr

*These authors contributed equally to this work.

(c) This is an open-access article distributed under the terms of the Creative Commons Attribution Non-Commercial License (http://creativecommons.org/ licenses/by-nc/4.0/), which permits unrestricted non-commercial use, distribution, and reproduction in any medium, provided the original work is properly cited.

Copyright (c) 2018 by the Korean Society for Stem Cell Research

\section{Introduction}

Recovery from spinal cord injury (SCI) is more difficult than from peripheral nerve damage, often leaving patients with permanent disabilities. SCIs can be time-consuming, psychologically and economically damaging disasters. The major causes of disability due to SCI involve loss of motor, sensory and autonomic nerves below the site of upper spinal injury. The severity of damage is classified as either complete or partial loss of function $(1,2)$. The severity of SCIs is determined not only by the primary cause but also by secondary processes such as ischemia, anoxia, free radical formation, and excitotoxicity that occur in the next few 
hours or days following injury (3). Several animal models have been used to refine clinical approaches to treat SCI and are categorized into two major groups based on the method of injury, transection models and contusion models. The contusion model is considered more clinically relevant than the transection model because SCI in the majority of patients is usually contusive (4). However, complete functional recovery from SCI is still not possible with current therapeutic approaches.

Transplantation of stem cells in rodent models of SCI has been shown to induce axonal growth, replace neuronal populations lost by injury, and improve functional recovery (reviewed in 5, 6). Human mesenchymal stem cells (hMSCs) derived from bone marrow are capable of self-renewing in vitro. These multipotent stem cells can differentiate into a variety of mesodermal cells $(7,8)$. Several research groups, including ours, have reported that hMSCs may also transdifferentiate into neuron-like cells that have neural cell markers and similar functional activity to that of neural cells (9-13). Manipulation of specific factors can accelerate transdifferentiation of hMSCs into neuron-like cells (14).

Oligodendrocyte lineage transcription factor 2 (Olig2) is, as a basic helix-loop-helix transcription factor, an essential regulatory factor for the differentiation of motor neurons and oligodendrocytes in the developing central nervous system $(15,16)$. Recent studies have demonstrated that Olig2 expression not only promotes neural stem cell differentiation into mature oligodendrocytes, but also improves locomotor recovery in rat spinal cord following contusive injury $(17,18)$. In this study, we genetically modified hMSCs to express Olig2 and investigated the effects of transplantation of Olig2-expressing hMSCs in a contusion model of rat spinal cord injury. Cells were transplanted in animals at 7 days after SCI, and behavioral outcomes and histological changes were evaluated 8 weeks after SCI.

\section{Materials and Methods}

\section{Human mesenchymal stem cell culture}

Cryopreserved adult hMSCs (Poietics ${ }^{\circledR}$ normal human mesenchymal stem cells) were purchased from Cambrex Bio Science Walkersville (Walkersville, MD, USA). We cultured hMSCs (passages 4-12) in Dulbecco's modified Eagle's medium (DMEM)-low glucose (Hyclone) containing $10 \%$ fetal bovine serum (FBS; Gibco-BRL) at $37^{\circ} \mathrm{C}$ with $5 \% \mathrm{CO}_{2}$.

\section{Lentiviral vector construction and virus production}

The lentiviral vector for expressing the mouse Olig2 gene was constructed by inserting the $E c o \mathrm{R} \mathrm{I}-E c o \mathrm{R} \mathrm{V}$ gene fragment into the $E c o \mathrm{R} \mathrm{I}$ - pme I site of the LentiM1.41 vector. The LentiM1.41 lentiviral vector was designed to produce interesting gene promotion from the murine cytomegalovirus (mCMV) promoter and to express eGFP from the PGK promoter. The nucleotide sequences of the constructs were verified by sequencing.

Macrogen, Inc. produced pseudotyped lentiviruses as described previously $(19,20)$. Briefly, three plasmids, a transfer vector, a VSV-G expression vector, and a gag-pol expression vector, were co-transfected into HEK-293T cells at a 1:1:1 molar ratio using Lipofectamine Plus (Invitrogen). The culture supernatant containing viral vector particles was harvested 48 hours later, clarified with a $0.45-\mu \mathrm{m}$ membrane filter (Nalgene), concentrated using a Centricon Plus-20 (Millipore) and immediately stored at $-70^{\circ} \mathrm{C}$ in a deep-freezer. Titers were determined by $\mathrm{p} 24$ ELISA and infection into HeLa cells. The eGFP expression of transduced cells was observed and photographed under a fluorescence microscope. The titer was approximately $10^{6} \sim 10^{7}$ transduction units (TU) per $\mathrm{mL}$ without further concentration.

\section{Lentiviral transduction}

All plasmid constructs were introduced into hMSCs by viral infection. The cells $\left(2.4 \times 10^{4}\right.$ cells/well $)$ were plated in six-well plates 2 days before transduction. hMSCs were infected with lentiviral vectors in the presence of $8 \mu \mathrm{g} / \mathrm{mL}$ polybrene. After 6 hours, the media were replaced with fresh growth media. Two days after viral infection, cells were selected using $1 \mu \mathrm{g} / \mathrm{mL}$ puromycin for 7 days.

\section{Immunocytochemistry}

Cells were fixed on coverslips with $4 \%$ paraformaldehyde at room temperature for 30 minutes and permeabilized for 10 minutes with $0.2 \%$ Triton $\mathrm{X}-100$ and $1 \%$ bovine serum albumin (BSA) in PBS. Blocking was performed by incubating cells for 1 hour with $5 \%$ normal goat serum in PBS. Cells were then incubated overnight at $4^{\circ} \mathrm{C}$ with rabbit polyclonal anti-Olig2, 1:5,000 (gift from Dr. C. Stiles, Harvard Medical School). After washing with PBS, cells were incubated with goat anti-rabbit conjugated with Alexa Fluor 546, 1:500 (Invitrogen). All cells were mounted with fluorescent mounting medium (Dako). Immunofluorescence was examined using a laser-scanning confocal microscope (Fluoview FV300, Olympus). 


\section{Spinal cord contusion injury}

Adult male Sprague-Dawley rats (Daehan Biolink, Chungbuk, Korea) weighing 260 280 g at the time of surgery were housed in groups of four and allowed free access to food and water. All animal procedures were carried out under the approval of the Institutional Animal Care and Use Committee of Seoul National University. Acute SCI was induced with an NYU Impactor. The rats were anesthetized by intraperitoneal injections of Zoletil $(35 \mathrm{mg} / \mathrm{kg})$ and Rompun $(2 \mathrm{mg} / \mathrm{kg})$ and a laminectomy was performed at the level of T9. The exposed dorsal surface of the spinal cord in each rat was then subjected to a weight-drop impact. To obtain moderately contused SCI models, a 10-g weight-impact rod was dropped from a $25-\mathrm{mm}$ height. The contusion impact velocity and compression rate were monitored to guarantee consistency of injury between experimental animals. During recovery, the rats' rectal temperatures were maintained at $37^{\circ} \mathrm{C}$ using a feedback- regulated heating pad. Postoperative nursing care included bladder expression twice per day. Gentamicin sulfate (1 $\mathrm{mg} / \mathrm{kg}$ ) was administered prophylactically to all animals for a week after SCI.

\section{Behavioral assessment after spinal cord injury}

Open-field testing procedures, also known as Basso-
Beattie-Bresnahan (BBB) procedures (21), were used to measure the functional recovery of rat hindlimbs. The scale used to measure hindlimb function with these procedures ranges from a score of 0 , indicating no spontaneous movement, to a maximum score of 21 , with an increasing score indicating the use of individual joints, coordinated joint movement, coordinated limb movement, weight-bearing and other functions. Rats were first gently adapted to the open field used for the test. After each rat had walked continuously in the open field, two investigators conducted 5-minute testing sessions on each leg. The open-field test was performed at least once per week from day 1 after SCI to 9 weeks after laminectomy on all animals in the study.

\section{Cell transplantation}

After rats had been subjected to behavioral tests for 1 week, they were randomly allocated into groups that received cell-culture medium, GFP-expressing hMSC transplants, or Olig2-GFP-expressing hMSC transplants. At 7 days after SCI, the rats were anesthetized by intraperitoneal injections of Zoletil $(35 \mathrm{mg} / \mathrm{kg}$ ) and Rompun $(2 \mathrm{mg} / \mathrm{kg}$ ), and received either $5 \mu \mathrm{L}$ of GFP-hMSCs $\left(1 \times 10^{5} / \mu \mathrm{L}\right)$ or Olig2-GFP-hMSCs $\left(1 \times 10^{5} / \mu \mathrm{L}\right)$ transplanted through a capillary glass tube into the epicenter
A
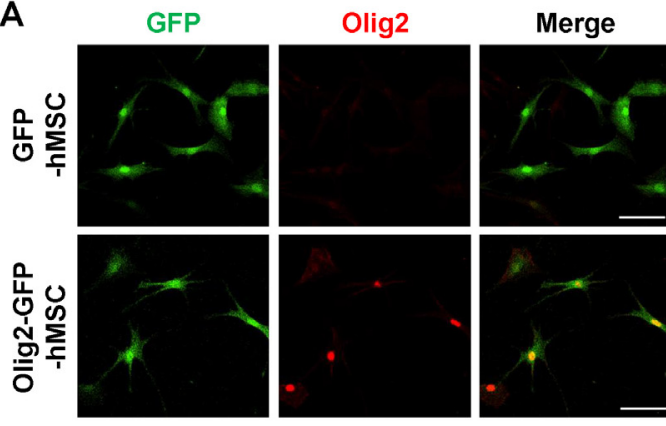

B

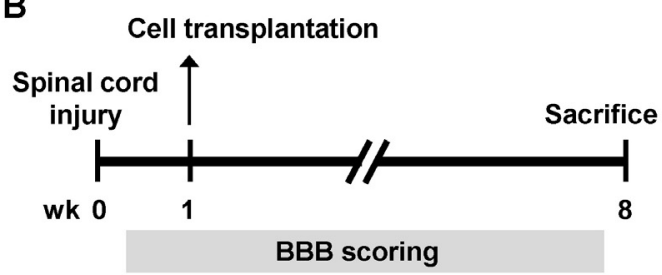

C

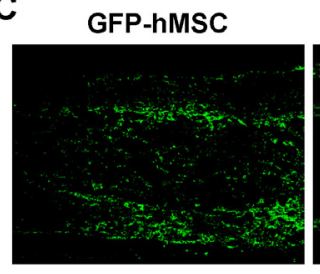

Fig. 1. Survival and distribution of Olig2-GFP-hMSCs in spinal cord lesion after contusive SCI. (A) Green fluorescent protein (GFP) and Olig2 expression in genetically modified hMSCs. The GFP-infected (GFP-hMSCs) and Olig2-GFP-infected (Olig2-GFP-hMSCs) hMSCs were immunostained with an anti-Olig2 (red) antibody. Colocalization of Oilg2 and GFP in the nucleus of Olig2-GFP-hMSCs is shown in the merged image (yellow). Scale bars: $100 \mu \mathrm{m}$. (B) Schematic diagram of the experimental design. (C) Immunohistochemistry of transplanted hMSCs in spinal cord lesion at 7 weeks after transplantation. Longitudinal spinal cord sections were immunostained for GFP at 7 weeks after transplantation of GFP-hMSCs and Olig2-GFP-hMSCs. Scale bars: $500 \mu \mathrm{m}$. The left side is rostral and the superior side is dorsal. 


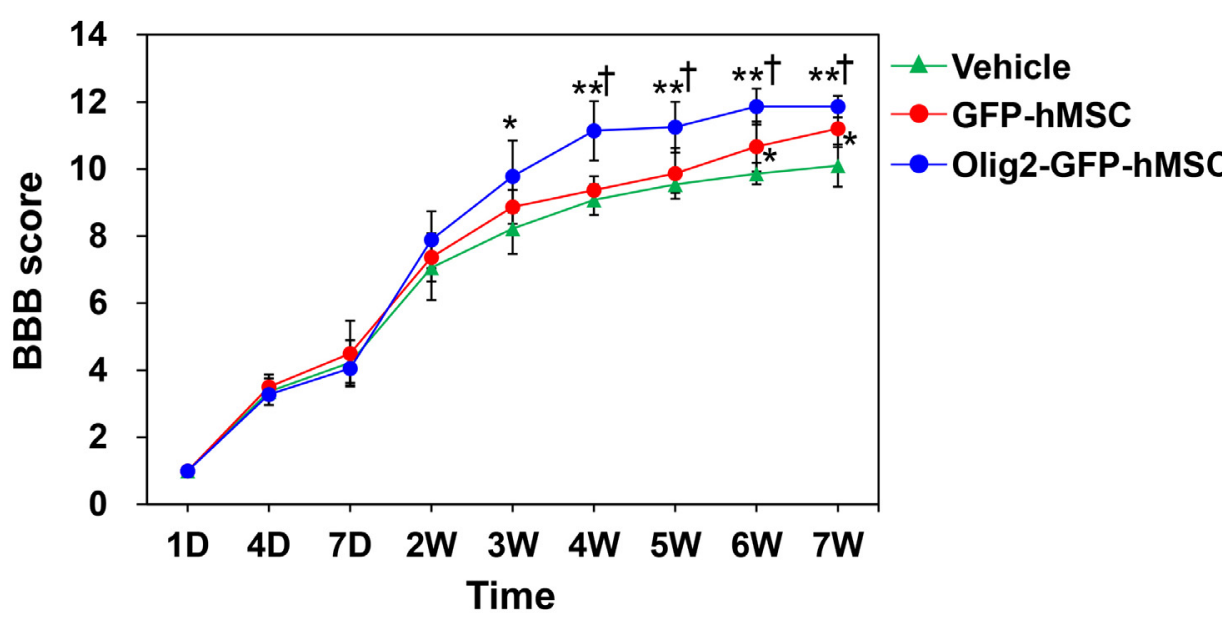

Fig. 2. Behavioral analysis of locomotor function. Effect of transplantation of Olig2-GFP-hMSCs on functional recovery after SCl. BBB locomotor scores of rats with $\mathrm{SCl}$ before and after transplantation of vehicle $(n=5)$, GFP-hMSCs $(n=5)$ and Olig2-GFP-hMSCs $(n=6)$ were analyzed. Arrow indicates the time point when transplantation was performed. Data are shown as mean \pm SEM. ${ }^{*} p$ $<0.05$ vs. vehicle transplant group, ${ }^{\dagger} \mathrm{p}<0.05$ vs. GFP-hMSC transplant group. of the injury, or received a $5-\mu \mathrm{L}$ injection of culture medium into the epicenter of the injury (control). To induce immunosuppression, cyclosporine A ( $1 \mathrm{mg} / 100 \mathrm{~g}$ ) was injected daily from 2 days before injury until the day of transplantation or injection of culture medium.

\section{Histological processing and immunohistochemistry}

At 8 weeks after the induction of SCI, all rats were sacrificed by perfusion with $4 \%$ paraformaldehyde in phosphate-buffered saline (PBS) under anesthesia. The spinal cords were removed and fixed for 18 hours in 4\% paraformaldehyde, followed by overnight immersion in 30\% sucrose in PBS. Serial longitudinal 12- $\mu$ m-thick sections of the spinal cord were obtained with a cryostat. The specimens were stored at $-80^{\circ} \mathrm{C}$ before further processing. Staining with hematoxylin and eosin (H\&E) was performed for histopathological analysis.

For immunohistochemical analysis, the spinal cord sections were rinsed with PBS for 3 minutes and fixed for 30 minutes with $4 \%$ paraformaldehyde. The sections were then treated with blocking solution to prevent nonspecific staining and were incubated with anti-neurofilament-M (1:5,000; rabbit polyclonal; Chemicon), anti-glial fibrillary acidic protein (GFAP) (1:1,000; mouse monoclonal; Chemicon), anti-Ibal (1:1,000; rabbit polyclonal; Wako), anti-Olig1 (1:20,000; rabbit polyclonal; kindly provided by Dr. C. Stiles, Harvard Medical School). After washing with PBS, the spinal cord sections were incubated with the following secondary antibodies: Alexa Fluor 488 anti-mouse IgG (Invitrogen) and Alexa Fluor 546 anti-rabbit IgG (Invitrogen). The sections were mounted on glass slides with a fluorescent mounting medium (Vectashield; Vector Laboratories) and observed under a laser-scanning confocal microscope (Fluoview FV300, Olympus).

\section{Histological analysis of tissue injury}

Histological analysis was carried out on $12-\mu \mathrm{m}$-thick longitudinal sections of spinal cord obtained from culture medium ( $\mathrm{n}=5)$, GFP-hMSCs $(\mathrm{n}=5)$, or Olig2-GFP-hMSCs $(n=6)$ transplanted rats that were stained with $\mathrm{H} \& \mathrm{E}$. Lesioned areas were outlined using Image Pro software and lesion sizes were calculated for statistical analysis. Serial sections were also immunohistochemically processed for each marker protein.

\section{Statistical analysis}

Data were analyzed by two-tailed Student's t-test or one-way analysis of variance (ANOVA) followed by post hoc comparisons with Fisher's protected least significant difference (PLSD) test. A probability value of $<0.05$ was considered statistically significant. All values were expressed as the mean \pm standard error of the mean (SEM).

\section{Results}

\section{Spinal cord injury and cell transplantation}

In this study, we ectopically expressed Olig2 in hMSCs using Olig2-GFP-containing lentiviral vectors (Olig2-GFPhMSCs). Control hMSCs were infected with GFP-containing lentiviral vectors (GFP-hMSCs). Both GFP-hMSCs and Olig2-GFP-hMSCs expressed green fluorescence under a fluorescence microscope (Fig. 1A). Immunostaining for Olig2 demonstrated that Olig2-GFP-hMSCs ectopically expressed Olig2, whereas GFP-hMSCs did not. No significant differences in morphology or viability were observed between these cells. We next sought to determine survival and distribution of GFP-hMSCs and Olig2-GFPhMSCs in spinal cord lesions at 7 weeks after transplantation. Contusion injuries of the rat thoracic spinal cord was performed according to a standard protocol (21). 
A

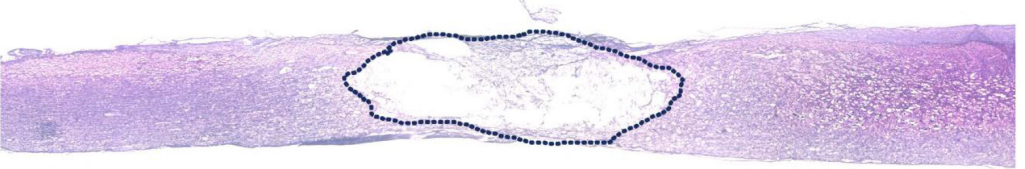

B

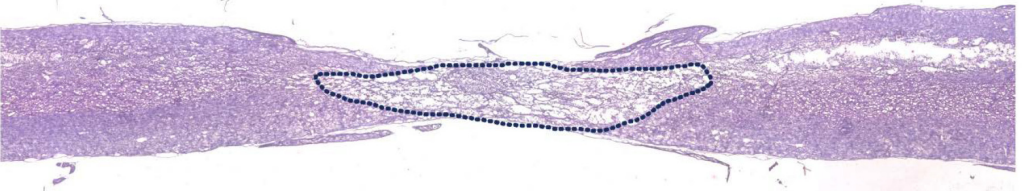

C

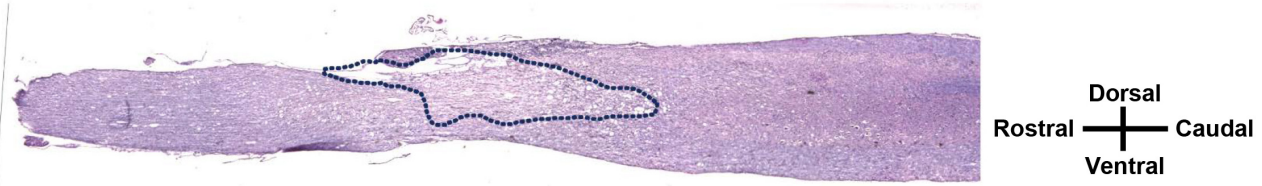

D

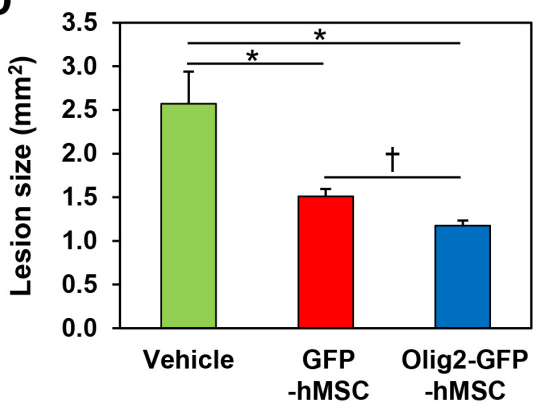

E

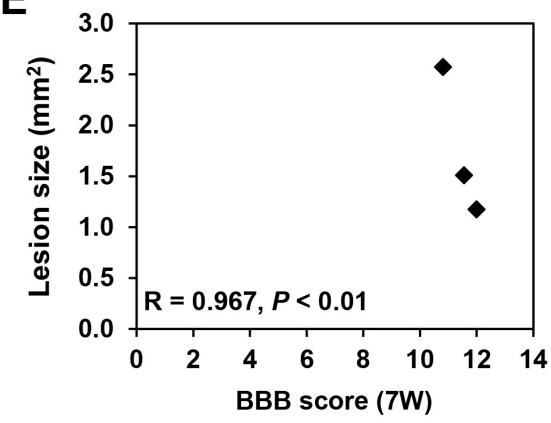

Fig. 3. Morphometry of spinal cord lesion after contusion SCl. Longitudinal spinal cord sections from the vehicle transplant group (A), GFP-hMSC transplant group (B), and Olig2-GFP-hMSC transplant group (C) were stained with hematoxylin and eosin (H\&E) at 8 weeks after $\mathrm{SCl}$. Histological analyses were conducted of longitudinal sections encompassing the lesion area. Dotted line outlines the lesion boundary. (D) The histograms represent measurements of the lesion area of vehicle transplant group, GFP-hMSC transplant group and Olig2-GFP-hMSC transplant group. (E) Correlations between BBB score 7 weeks post-injury and lesion size in the spinal cord tissue. Data are shown as mean \pm SEM. The results are representative of at least three rats in each group. ${ }^{*} p<0.05$ vs. vehicle transplant group, ${ }^{\dagger} p<0.05$ vs. GFP-hMSC transplant group.

At 7 days after SCI, animals received transplantation of GFP-hMSCs or Olig2-GFP-hMSCs (Fig. 1B). Transplanted GFP-hMSCs and Olig2-GFP-hMSCs were found by GFP immunohistochemistry in the spinal cord lesion at 7 weeks after cell transplantation (Fig. 1C), indicating survival of engrafted cells.

\section{Behavioral analysis of locomotor function}

After the induction of SCI, all experimental rats showed flaccid paralysis of hindlimbs during the first few days after surgery. To determine the effect of Olig2-GFP-hMSC transplantation on functional recovery in contusion SCI rats, BBB scores were evaluated one day after injury. As shown in Fig. 2, one week after SCI (the day before hMSC transplantation), all rats received a score of 5 or less. There were no significant differences among the three groups. At the second week post-treatment, animals transplanted with Olig2-GFP-hMSCs showed significantly faster functional recovery. From 3 to 7 weeks following treatment, rats transplanted with Olig2-GFP-hMSCs showed significant increases in BBB scores compared to rats transplanted with GFP-hMSCs or sham-injected with culture medium (vehicle). At 6 and 7 weeks following treatment, the BBB scores of rats transplanted with GFP-hMSCs were greater than those of sham-injected rats. These results indicate that rats receiving transplantation of Olig2GFP-hMSCs exhibited significantly enhanced functional recovery compared to rats transplanted with GFP-hMSCs or sham-injected with vehicle.

\section{Histological analysis}

To elucidate the mechanisms of enhanced locomotor re- 

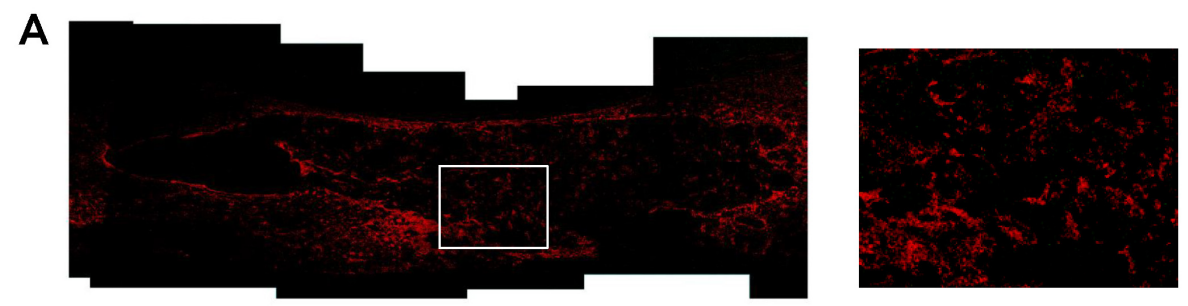

B
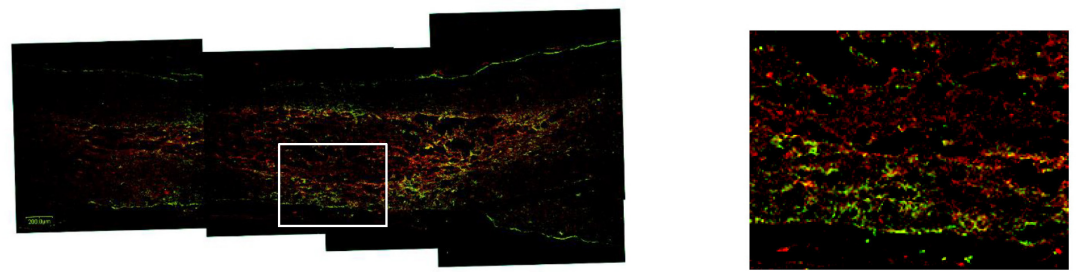

\section{C}
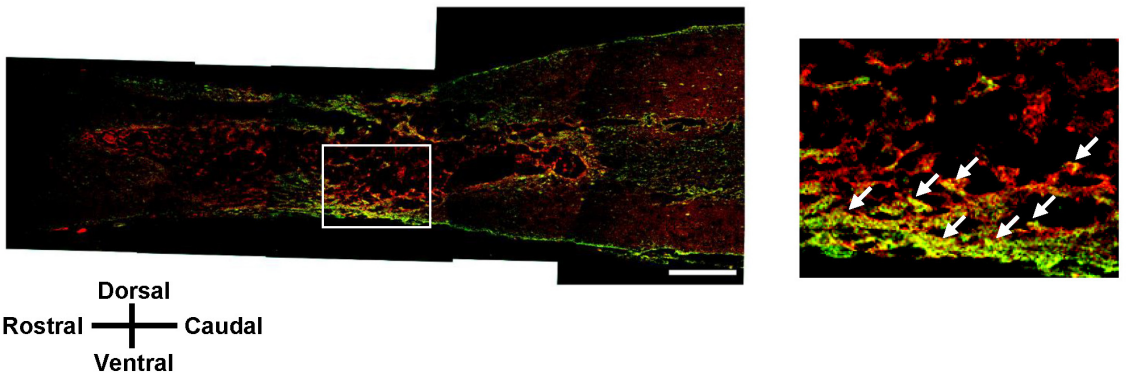

Fig. 4. Olig1 immunohistochemistry of the spinal cord lesion at 7 weeks after transplantation. Longitudinal spinal cord sections were immunostained for Olig1 and GFP 7 weeks after transplantation of vehicle (A), GFP-hMSCs (B) and Olig2-GFP-hMSCs (C). The right panel is magnified images of the boxed area. White arrows show colocalization of Olig1 to GFP-positive cells. Scale bars: $500 \mu \mathrm{m}$. In all images, the left side is rostral and the superior side is dorsal.

covery in rats transplanted with Olig2-GFP-hMSCs, we performed morphological analysis of spinal cord lesions using $H \& E$ staining. $H \& E$ staining showed that various cavity morphologies were formed at the spinal cord lesions of all SCI animals, including those that received transplantations of GFP-hMSCs or Olig2-GFP-hMSCs as well as vehicle (Fig. 3A-C). The spinal cord cavity was largest in the vehicle transplant group. The spinal cord cavity of the GFP-hMSC transplant group and the Olig2-GFPhMSC transplant group was smaller than that of the vehicle transplant group. H\&E stained images were manually outlined and analyzed to quantify cavity area (Fig. 3A-C). Cavity size in the Olig2-GFP-hMSC transplant group $\left(1.18 \pm 0.06 \mathrm{~mm}^{2}\right)$ was significantly reduced compared to the GFP-hMSC transplant group $(1.46 \pm 0.09$ $\left.\mathrm{mm}^{2}\right)$ as well as the vehicle transplant group $(2.57 \pm 0.37$ $\left.\mathrm{mm}^{2}\right) \quad(\mathrm{p}<0.05$, Fig. 3D). These results indicate that Olig2-GFP-hMSCs attenuate glial scar formation in spinal cord lesions. Correlation analysis showed a significant correlation $(\mathrm{r}=-0.967, \mathrm{p}<0.01)$ between the BBB score at 7 weeks post-spinal cord injury and the size of the spinal cord injury site (Fig. 3E).

\section{Immunohistochemical analysis}

At 7 weeks after SCI, we performed immunohistochemical analysis in longitudinal sections of injured spinal cord for all three treatment groups: vehicle transplantation, GFP-hMSC transplantation, and Olig2-GFP-hMSC transplantation. Oligodendroglial lineage-associated marker Olig1 immunohistochemistry showed that expression of GFP and Olig1 was higher in the Olig2-GFP-hMSC transplant group compared to the GFP-hMSC transplant group (Fig. 4). This suggests that transplanted Olig2-GFPhMSCs partially differentiated into Olig1-positive oligodendrocyte-like cells in spinal cords. Next, we investigated the expression of NF-M in transplanted GFP-hMSCs and host cells in the injured spinal cord. Transplanted Olig2-GFP-hMSCs, but not GFP-hMSCs, seemed to be directly associated with NF-M positive axons (Fig. 5). Furthermore, NF-M positive axons were more abundant in the Olig2-GFP-hMSC transplant group than in the GFP-hMSC transplant group. As expected, there were few 

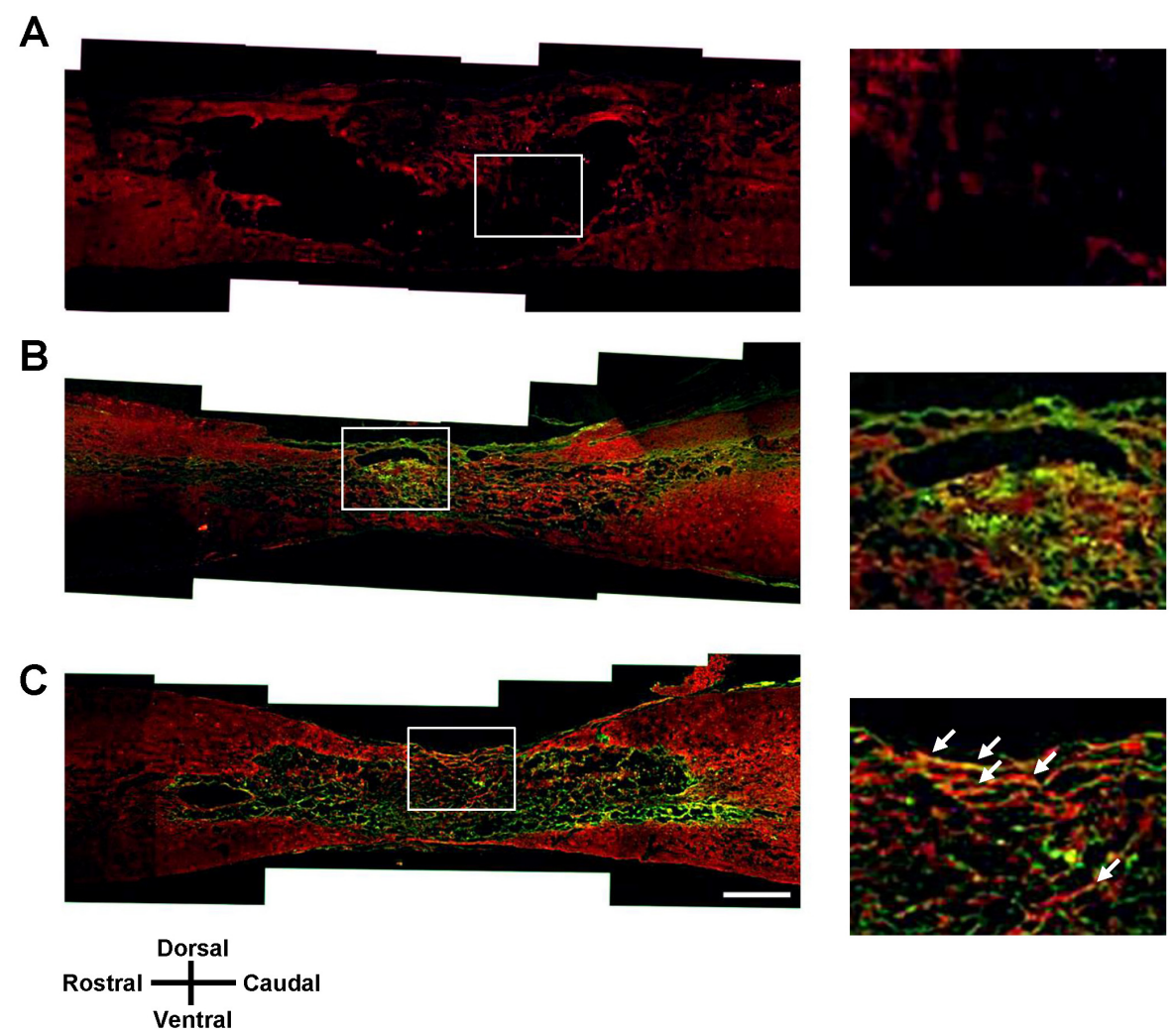

Fig. 5. NF-M immunohistochemistry of the spinal cord lesion at 7 weeks after transplantation. Longitudinal spinal cord sections were immunostained for NF-M and GFP 7 weeks after transplantation of vehicle (A), GFP-hMSCs (B), and Olig2-GFP-hMSCs (C). The right panel shows magnified images of the boxed area. White arrows show colocalization of NF-M to GFP-positive cells. Scale bars: $500 \mu \mathrm{m}$. In all images, the left side is rostral and the superior side is dorsal.

NF-M positive axons in the epicenters of injured spinal cords in the vehicle transplant group. Microglial marker Ibal immunohistochemistry revealed increased density of Ibal-positive microglial cells in the host tissue of the vehicle transplant group when compared to the Olig2-GFPhMSC transplant or GFP-hMSC transplant groups (Fig. 6). Next, we investigated the expression of astrocyte marker GFAP in transplanted hMSCs and host cells at the injured spinal cord. Quite a few cells expressed both GFP and GFAP in the spinal cord in the Olig2-GFP-hMSC transplant group (Fig. 7). This suggests that transplanted Olig2-GFP-hMSCs partially differentiated into Olig2- lineage astrocytes (22).

\section{Discussion}

In the present study, we demonstrate that the transplantation of hMSCs that are genetically modified to express Olig2 enhances locomotor function in a rat model of contusive SCI model. Previous studies showed that transplants of bone marrow-derived MSCs have ther- apeutic effects in SCI (23-26). Indeed, in the present study, transplantation of control hMSCs reduces cavity area in the injured spinal cord and leads to functional improvements in SCI. Although various types of stem cells have been used for the treatment of SCI, MSC transplantation has several advantages $(27,28)$ : MSCs are readily available from donors and can be expanded and transplanted autologously. MSCs are at very low risk for malignant transformation. In addition, autologous transplantation of MSCs is known to have immunosuppressive properties. The safety of MSCs has been demonstrated in several clinical studies $(29,30)$. However, it is necessary to optimize therapeutic applications to treat SCI using MSC transplantation in clinical practice. The present study indicated that transplantation of hMSCs genetically modified to express Olig2 enhances locomotor function more significantly than control hMSCs in a rat model of contusive SCI model.

Several in vivo models of SCI have been widely used to study chronic pathologies after damage to spinal nerves $(31,32)$. In spinal cord tissues adjacent to the injured 

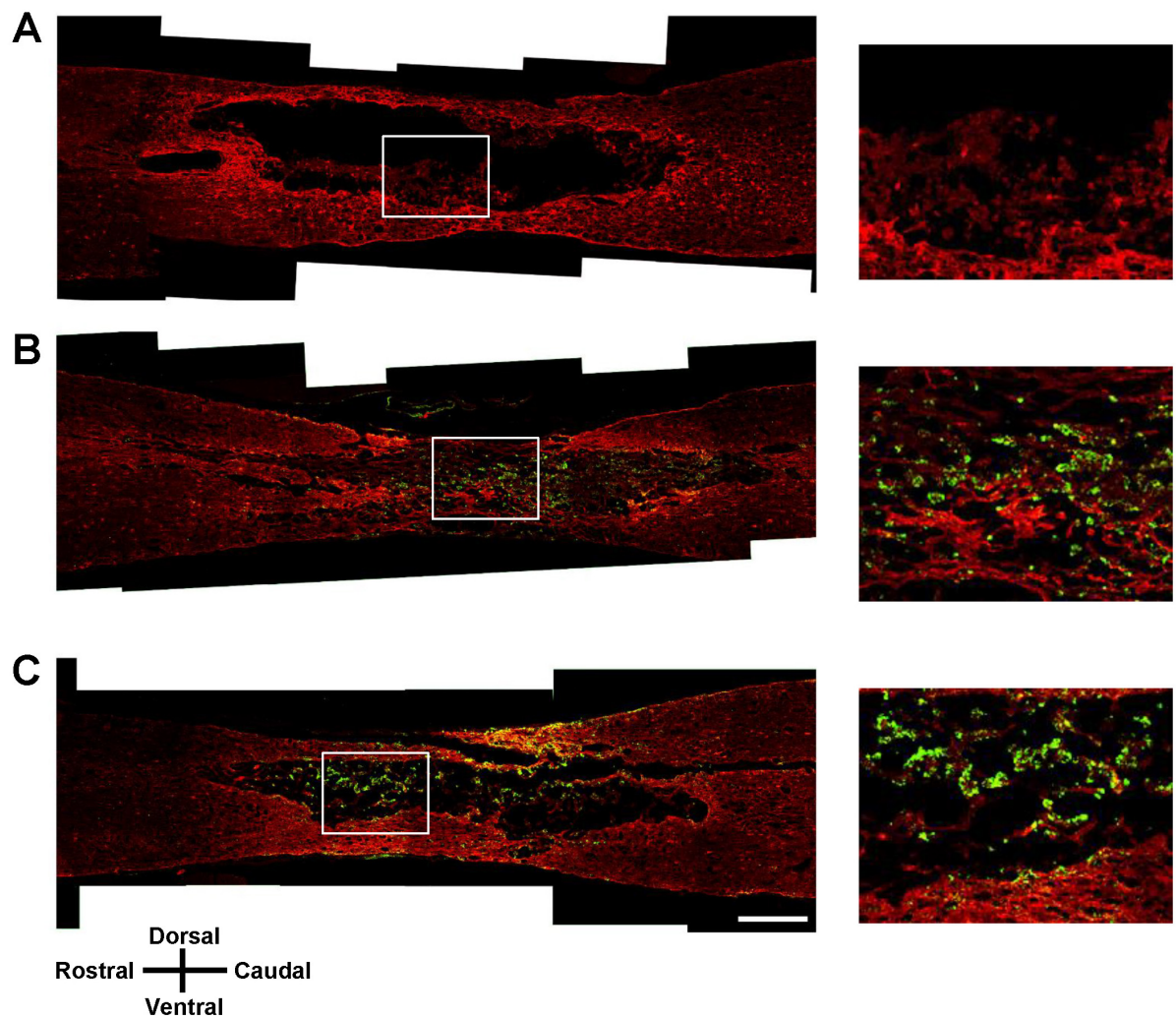

Fig. 6. Iba1 immunohistochemistry of the spinal cord lesion 7 weeks after transplantation. Longitudinal spinal cord sections were immunostained for Iba1 and GFP 7 weeks after transplantation of vehicle (A), GFP-hMSCs (B), and Olig2-GFP-hMSCs (C). The right panel shows magnified images of the boxed area. Scale bars: $500 \mu \mathrm{m}$. In all images, the left side is rostral and the superior side is dorsal.

area, direct injury and tissue disruption is alleviated as it moves away from the area of injury (33). SCI is associated with loss of neurons and glia as well as many changes in gene expression and protein levels, leading to demyelination (34). In this study, Olig2 expression enhanced the therapeutic effects of hMSCs to improve functional recovery after SCI, possibly by modulating the microenvironment. This effect may be due to the production of growth factors and neurotrophic factors that regulate neuronal development, cell survival, and neuritogenesis, but further studies are required to elucidate the precise mechanisms involved. Olig2 is highly expressed in the ventral spine during the early stages of human development and plays an important role in oligodendrocyte differentiation of the spine (35). We found that Olig1positive cells were the most abundant in injured spinal cord after transplantation of Olig2-GFP-hMSCs. In addition, some transplanted Olig2-GFP-hMSCs expressed Olig1 in the injured spinal cord, whereas control GFPhMSCs did not. Our results suggest that hMSCs that are genetically modified to express Olig2 can transdifferentiate into oligodendrocyte-like cells in the spinal cord, results that are supported by those of a previous study (36).

Collectively, our results suggest that the transplantation of Olig2-expressing hMSCs has the potential to improve functional recovery of injured spinal cord. BBB scores revealed that the transplantation of Olig2-expressing hMSCs resulted in greater functional improvement in SCI compared that observed in a control hMSC transplant group. Treatment using hMSCs that are genetically modified to express Olig2 is thus considered to be a safe and optimal therapy for SCI.

\section{Acknowledgements}

We would like to thank Hyun-Goo Kang and Su Jin Jeong for their critical reading of the manuscript. This research was supported by the Basic Science Research Program through the National Research Foundation of Korea (M-SC，2012R1A1A2007945, NRF-2015R1D1A1A 0105 6950, and H-WP, 2015R1A5A1009701) funded by the Ministry of Education, and by a grant of the Korean Health Technology R\&D Project (M-SC, HI17C2160), Ministry of Health \& Welfare, Republic of Korea. 

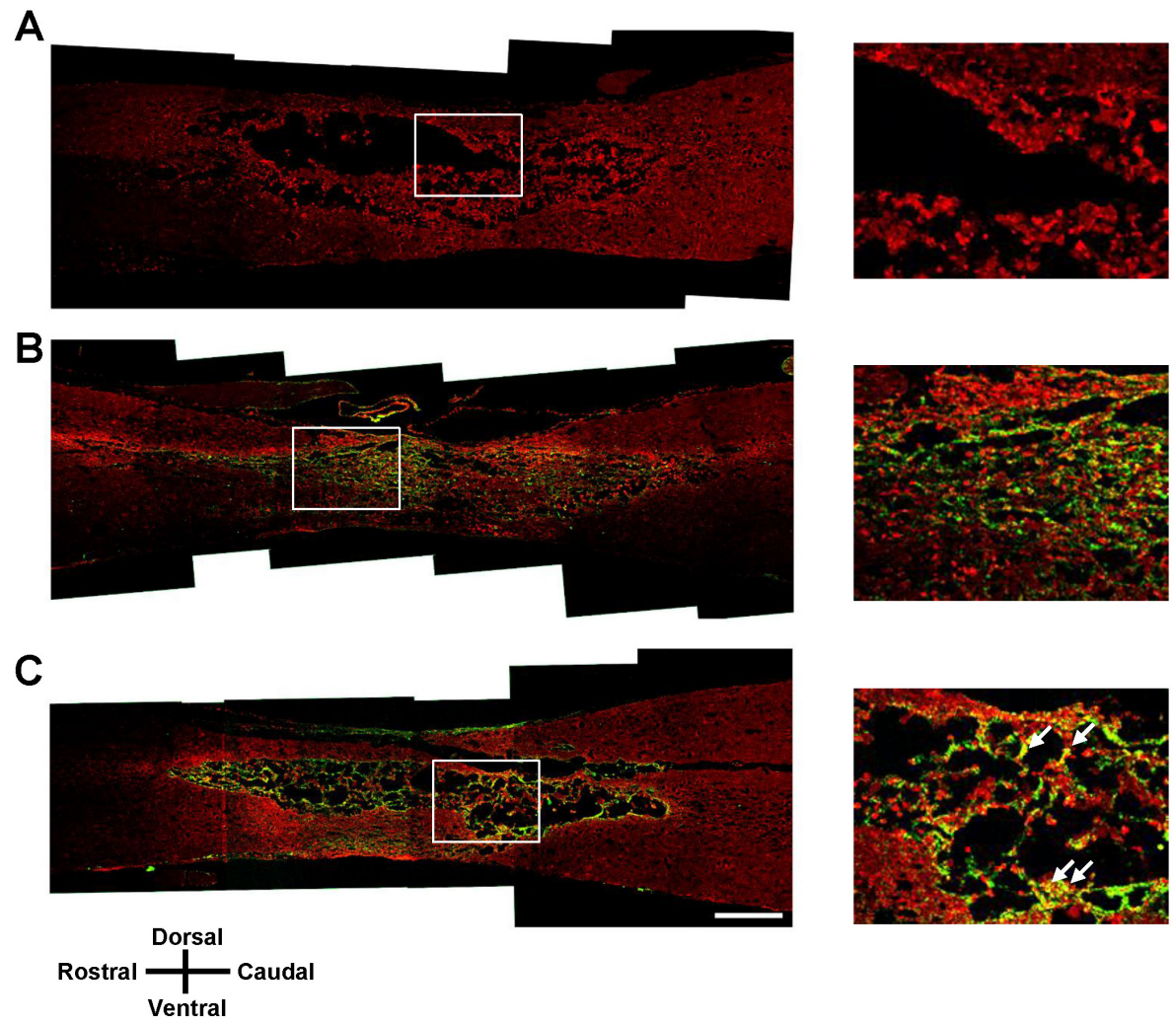

Fig. 7. GFAP immunohistochemistry of the spinal cord lesion at 7 weeks after transplantation. Longitudinal spinal cord sections were immunostained for GFAP and GFP 7 weeks after transplantation of vehicle (A), GFP-hMSCs (B), and Olig2-GFP-hMSCs (C). The right panel shows magnified images of the boxed area. White arrows show colocalization of GFAP to GFP-positive cells. Scale bars: $500 \mu \mathrm{m}$. In all images, the left side is rostral and the superior side is dorsal.

\section{Potential Conflict of Interest}

The authors have no conflicting financial interest.

\section{References}

1. Di Giovanni S. Regeneration following spinal cord injury, from experimental models to humans: where are we? Expert Opin Ther Targets 2006;10:363-376

2. Tator $\mathrm{CH}$, Fehlings MG. Review of the secondary injury theory of acute spinal cord trauma with emphasis on vascular mechanisms. J Neurosurg 1991;75:15-26

3. Beattie MS, Farooqui AA, Bresnahan JC. Review of current evidence for apoptosis after spinal cord injury. J Neurotrauma 2000;17:915-925

4. Kwon BK, Hillyer J, Tetzlaff W. Translational research in spinal cord injury: a survey of opinion from the SCI community. J Neurotrauma 2010;27:21-33

5. Nandoe Tewarie RS, Hurtado A, Bartels RH, Grotenhuis A, Oudega M. Stem cell-based therapies for spinal cord injury. J Spinal Cord Med. J Spinal Cord Med 2009;32:105114

6. Thuret S, Moon LD, Gage FH. Therapeutic interventions after spinal cord injury. Nat Rev Neurosci 2006;7:628-643
7. Bianco P. "Mesenchymal" stem cells. Annu Rev Cell Dev Biol 2014;30:677-704

8. Gardner OF, Alini M, Stoddart MJ. Mesenchymal stem cells derived from human bone marrow. Methods Mol Biol 2015;1340:41-52

9. Woodbury D, Schwarz EJ, Prockop DJ, Black IB. Adult rat and human bone marrow stromal cells differentiate into neurons. J Neurosci Res 2000;61:364-370

10. Aguilera-Castrejon A, Pasantes-Morales H, Montesinos JJ, Cortés-Medina LV, Castro-Manrreza ME, Mayani H, Ramos-Mandujano G. Improved proliferative capacity of NP-like cells derived from human mesenchymal stromal cells and neuronal transdifferentiation by small molecules. Neurochem Res 2017;42:415-427

11. Darabi S, Tiraihi T, Delshad A, Sadeghizadeh M, Taheri T, Hassoun HK. Creatine enhances transdifferentiation of bone marrow stromal cell-derived neural stem cell into GABAergic neuron-like cells characterized with differential gene expression. Mol Neurobiol 2017;54:1978-1991

12. Park HW, Cho JS, Park CK, Jung SJ, Park CH, Lee SJ, Oh SB, Park YS, Chang MS. Directed induction of functional motor neuron-like cells from genetically engineered human mesenchymal stem cells. PLoS One 2012;7:e35244

13. Park HW, Lim MJ, Jung H, Lee SP, Paik KS, Chang MS. 
Human mesenchymal stem cell-derived Schwann cell-like cells exhibit neurotrophic effects, via distinct growth factor production, in a model of spinal cord injury. Glia 2010;58: 1118-1132

14. Sugimori M, Nagao M, Bertrand N, Parras CM, Guillemot F, Nakafuku M. Combinatorial actions of patterning and HLH transcription factors in the spatiotemporal control of neurogenesis and gliogenesis in the developing spinal cord. Development 2007;134:1617-1629

15. Zhou Q, Wang S, Anderson DJ. Identification of a novel family of oligodendrocyte lineage-specific basic helix-loophelix transcription factors. Neuron 2000;25:331-343

16. Lu QR, Yuk D, Alberta JA, Zhu Z, Pawlitzky I, Chan J, McMahon AP, Stiles CD, Rowitch DH. Sonic hedgehog-regulated oligodendrocyte lineage genes encoding bHLH proteins in the mammalian central nervous system. Neuron 2000;25:317-329

17. Hu JG, Shen L, Wang R, Wang QY, Zhang C, Xi J, Ma SF, Zhou JS, Lü HZ. Effects of Olig2-overexpressing neural stem cells and myelin basic protein-activated T cells on recovery from spinal cord injury. Neurotherapeutics 2012;9: $422-445$

18. Hwang DH, Kim BG, Kim EJ, Lee SI, Joo IS, Suh-Kim H, Sohn S, Kim SU. Transplantation of human neural stem cells transduced with Olig2 transcription factor improves locomotor recovery and enhances myelination in the white matter of rat spinal cord following contusive injury. BMC Neurosci 2009;10:117

19. Dull T, Zufferey R, Kelly M, Mandel RJ, Nguyen M, Trono D, Naldini L. A third-generation lentivirus vector with a conditional packaging system. J Virol 1998;72:8463-8471

20. Follenzi A, Ailles LE, Bakovic S, Geuna M, Naldini L. Gene transfer by lentiviral vectors is limited by nuclear translocation and rescued by HIV-1 pol sequences. Nat Genet 2000;25:217-222

21. Basso DM, Beattie MS, Bresnahan JC. Graded histological and locomotor outcomes after spinal cord contusion using the NYU weight-drop device versus transection. Exp Neurol 1996;139:244-256

22. Tatsumi K, Isonishi A, Yamasaki M, Kawabe Y, MoritaTakemura S, Nakahara K, Terada Y, Shinjo T, Okuda H, Tanaka T, Wanaka A. Olig2-lineage astrocytes: a distinct subtype of astrocytes that differs from GFAP astrocytes. Front Neuroanat 2018;12:8

23. Dai G, Liu X, Zhang Z, Yang Z, Dai Y, Xu R. Transplantation of autologous bone marrow mesenchymal stem cells in the treatment of complete and chronic cervical spinal cord injury. Brain Res 2013;1533:73-79

24. Chen YB, Jia QZ, Li DJ, Sun JH, Xi S, Liu LP, Gao DX,
Jiang DW. Spinal cord injury in rats treated using bone marrow mesenchymal stem-cell transplantation. Int J Clin Exp Med 2015;8:9348-9354

25. Torres-Espín A, Redondo-Castro E, Hernández J, Navarro $\mathrm{X}$. Bone marrow mesenchymal stromal cells and olfactory ensheathing cells transplantation after spinal cord injury--a morphological and functional comparison in rats. Eur J Neurosci 2014;39:1704-1717

26. Yin F, Guo L, Meng CY, Liu YJ, Lu RF, Li P, Zhou YB. Transplantation of mesenchymal stem cells exerts anti-apoptotic effects in adult rats after spinal cord ischemiareperfusion injury. Brain Res 2014;1561:1-10

27. English K. Mechanisms of mesenchymal stromal cell immunomodulation. Immunol Cell Biol 2013;91:19-26

28. Stagg J, Galipeau J. Mechanisms of immune modulation by mesenchymal stromal cells and clinical translation. Curr Mol Med 2013;13:856-867

29. Weiss DJ, Casaburi R, Flannery R, LeRoux-Williams M, Tashkin DP. A placebo-controlled, randomized trial of mesenchymal stem cells in COPD. Chest 2013;143:15901598

30. Karussis D, Karageorgiou C, Vaknin-Dembinsky A, Gowda-Kurkalli B, Gomori JM, Kassis I, Bulte JW, Petrou P, Ben-Hur T, Abramsky O, Slavin S. Safety and immunological effects of mesenchymal stem cell transplantation in patients with multiple sclerosis and amyotrophic lateral sclerosis. Arch Neurol 2010;67:1187-1194

31. Casha S, Yu WR, Fehlings MG. FAS deficiency reduces apoptosis, spares axons and improves function after spinal cord injury. Exp Neurol 2005;196:390-400

32. Krassioukov AV, Johns DG, Schramm LP. Sensitivity of sympathetically correlated spinal interneurons, renal sympathetic nerve activity, and arterial pressure to somatic and visceral stimuli after chronic spinal injury. J Neurotrauma 2002;19:1521-1529

33. Buss A, Schwab ME. Sequential loss of myelin proteins during Wallerian degeneration in the rat spinal cord. Glia 2003;42:424-432

34. Di Giovanni S, De Biase A, Yakovlev A, Finn T, Beers J, Hoffman EP, Faden AI. In vivo and in vitro characterization of novel neuronal plasticity factors identified following spinal cord injury. J Biol Chem 2005;280:2084-2091

35. Jakovcevski I, Zecevic N. Olig transcription factors are expressed in oligodendrocyte and neuronal cells in human fetal CNS. J Neurosci 2005;25:10064-10073

36. Zhang HT, Fan J, Cai YQ, Zhao SJ, Xue S, Lin JH, Jiang $\mathrm{XD}, \mathrm{Xu}$ RX. Human Wharton's jelly cells can be induced to differentiate into growth factor-secreting oligodendrocyte progenitor-like cells. Differentiation 2010;79:15-20 